\title{
Employing Unnatural Amino Acids in the Preparation of Bioconjugates
}

\author{
Johnathan C. Maza \\ Taylor H. Jacobs \\ Diya M. Uthappa \\ Douglas D. Young* \\ Synlett 2016, 27, 805.
}

The title has been corrected for the final online and print versions. 